PHYSICAL REVIEW D 96, 089901(E) (2017)

\title{
Erratum: Parametrized post-Einsteinian framework for gravitational wave bursts [Phys. Rev. D 90, 104010 (2014)]
}

\author{
Nicholas Loutrel, Nicolás Yunes, and Frans Pretorius \\ (Received 9 August 2017; published 5 October 2017)
}

DOI: 10.1103/PhysRevD.96.089901

In this erratum, we correct certain elements of the parametrized post-Einsteinian (ppE) model for the evolution of gravitational wave $(\mathrm{GW})$ bursts from highly eccentric binary systems in time-frequency space presented in the original paper. As part of the computation of a ppE burst model, we considered an effective-one-body description for binary systems with generic modifications to the Newtonian potential. We claimed that the Newtonian potential in a generic modified theory of gravity can be written as shown in Eq. (B1). While the derivations in Appendix B are self-consistent, this scaling of the corrections is not suitable for some modified theories, and in particular does not give the proper result for the Einsteindilaton-Gauss-Bonnet (EdGB) gravity [1] example presented in the original paper. To make the model apply to such theories, one must model the corrections to the Newtonian kinetic energy and gravitational potential via

$$
\delta T=\frac{1}{2} \mu\left[r^{2} \dot{\phi}^{2} \tilde{\beta}\left(\frac{M}{r}\right)^{\tilde{b}}+\dot{r}^{2} \tilde{\gamma}\left(\frac{M}{r}\right)^{\tilde{c}}\right], \quad \delta U=-\frac{\mu M}{r}\left[\tilde{\alpha}\left(\frac{M}{r}\right)^{\tilde{a}}\right]
$$

where $M$ is the total mass of the binary, $\mu$ is the reduced mass, $(\tilde{\alpha}, \tilde{\beta}, \tilde{\gamma})$ are amplitude parameters that depend on the coupling constants of the theory, and $(\tilde{a}, \tilde{b}, \tilde{c}) \in \mathbb{R}$ that control the post-Newtonian (PN) order of the modifications. This expression corrects Eq. (B1) by (i) including an additional term proportional to $\dot{r}$, where $(\tilde{\gamma}, \tilde{c})$ are not related to the modification to the energy flux due to GW emission given in Eq. (29) of the original paper, and (ii) making the terms proportional to $(\tilde{\alpha}, \tilde{\beta}, \tilde{\gamma})$ depend on $(M / r)$ instead of $\left(M / r_{p}\right)$, where $r_{p}$ is the pericenter distance of the binary.

These modifications to the Lagrangian now propagate to various other equations and introduce additional corrections to the original paper, which we list below:

(i) The conserved orbital energy and angular momentum written in terms of the pericenter distance for bound orbits takes the form $E=E_{\mathrm{N}}+\delta E$ and $L=L_{\mathrm{N}}+\delta L$, with

$$
\begin{aligned}
\frac{\delta E}{E_{\mathrm{N}}} & =-\frac{1}{2} \tilde{\alpha}\left(\frac{M}{r_{p}}\right)^{\tilde{a}} \mathcal{G}(e ; \tilde{a}, 0,1)-\frac{1}{4} \tilde{\beta}\left(\frac{M}{r_{p}}\right)^{\tilde{b}} \mathcal{G}(e ; \tilde{b}, 1,1), \\
\frac{\delta L}{L_{\mathrm{N}}} & =\frac{1}{4} \tilde{\alpha}\left(\frac{M}{r_{p}}\right)^{\tilde{a}} \mathcal{G}(e ; \tilde{a}, 1,0)+\frac{1}{8} \tilde{\beta}\left(\frac{M}{r_{p}}\right)^{\tilde{b}} \mathcal{G}(e ; \tilde{b}, 2,0),
\end{aligned}
$$

the Newtonian $\left(E_{\mathrm{N}}, L_{\mathrm{N}}\right)$ given in Eqs. (5.2) and (5.3) in [2], and

$$
\mathcal{G}(e ; q, m, n)=\frac{(1+e)^{m}(1-e)^{n}}{e}\left[1-\left(\frac{1-e}{1+e}\right)^{q+m-n}\right]
$$

These deformations correct Eqs. (27) and (28).

(ii) The orbital period of bound orbits becomes $T_{\mathrm{orb}}=T_{\mathrm{orb}}^{\mathrm{N}}+\delta T_{\mathrm{orb}}$, where $T_{\mathrm{orb}}^{\mathrm{N}}$ is given by Eq. (16) in [1] and

$$
\frac{\delta T_{\text {orb }}}{T_{\mathrm{orb}}^{\mathrm{N}}}=\frac{1}{8 \pi} \tilde{\alpha}\left(\frac{M}{r_{p}}\right)^{\tilde{a}} \mathcal{P}_{1}(e ; \tilde{a}, 1)+\frac{1}{16 \pi} \tilde{\beta}\left(\frac{M}{r_{p}}\right)^{\tilde{b}} \mathcal{P}_{1}(e ; \tilde{b}, 2)+\frac{1}{4 \pi} \tilde{\gamma}\left(\frac{M}{r_{p}}\right)^{\tilde{c}} \mathcal{P}_{2}(e),
$$

with 


$$
\begin{gathered}
\mathcal{P}_{1}(e ; p, n)=\frac{\left(1-e^{2}\right)^{3 / 2}}{e^{2}(1+e)^{p}} \int_{0}^{2 \pi} \frac{d \psi}{s^{2}(1+e c)^{2}}\left[(1-e)^{p+n}(1-c)(2+e+e c)\right. \\
\left.+(1+e)^{p+n}(1+c)(2-e+e c)-4(1+e c)^{p+n}\right], \\
\mathcal{P}_{2}(e)=\frac{\left(1-e^{2}\right)^{3 / 2}}{(1+e)^{\tilde{c}}} \int_{0}^{2 \pi} d \psi(1+e c)^{\tilde{c}-2},
\end{gathered}
$$

and $(c, s)=(\cos \psi, \sin \psi)$ with $\psi$ the true anomaly. This corrects Eq. (B12) in Appendix B.

(iii) The pericenter velocity for bound orbits becomes $v_{p}=v_{p}^{\mathrm{N}}+\delta v_{p}$, where $v_{p}^{\mathrm{N}}=\left[M(1+e) / r_{p}\right]^{1 / 2}$ and

$$
\frac{\delta v_{p}}{v_{p}^{\mathrm{N}}}=\frac{1}{4} \tilde{\alpha}\left(\frac{M}{r_{p}}\right)^{\tilde{a}} \mathcal{V}_{\alpha}(e)-\frac{1}{8} \tilde{\beta}\left(\frac{M}{r_{p}}\right)^{\tilde{b}} \mathcal{V}_{\beta}(e)
$$

with

$$
\mathcal{V}_{\alpha}(e)=1+\left(\frac{1-e}{1+e}\right)^{\tilde{a}}+\frac{1}{e}\left[1-\left(\frac{1-e}{1+e}\right)^{\tilde{a}}\right], \quad \mathcal{V}_{\beta}(e)=4+\left[2-\left(\frac{1+e^{2}}{e}\right)\right]\left[1-\left(\frac{1-e}{1+e}\right)^{\tilde{b}}\right]
$$

This corrects Eq. (B14) in Appendix B of the original paper.

(iii) The mappings between $\mathrm{ppE}$ deformations given in Eqs. (27)-(30) and the ppE parameters that define the deformations to the burst model, specifically $\delta \lambda^{a}=\left(\alpha_{\mathrm{ppE}}, \beta_{\mathrm{ppE}}, \gamma_{\mathrm{ppE}}, \delta_{\mathrm{ppE}}\right)$ and $\delta \ell^{a}=\left(\bar{a}_{\mathrm{ppE}}, \bar{b}_{\mathrm{ppE}}, \bar{c}_{\mathrm{ppE}}, \bar{d}_{\mathrm{ppE}}\right)$, are corrected to

$$
\alpha_{\mathrm{ppE}}\left(\frac{M}{r_{p}}\right)^{\bar{a}_{\mathrm{ppE}}}=\mathrm{LO}\left[\frac{\delta T_{\mathrm{orb}}}{T_{\mathrm{orb}}^{\mathrm{N}}}\right], \quad \beta_{\mathrm{ppE}}\left(\frac{M}{r_{p}}\right)^{\bar{b}_{\mathrm{ppE}}}=\mathrm{LO}\left[\frac{\delta v_{p}}{v_{p}^{\mathrm{N}}}\right]
$$

where $\delta T_{\text {orb }}$ and $\delta v_{p}$ are given in Eqs. (5) and (8), respectively. These correct Eqs. (44) and (45).

(iv) The mapping between the Lagrangian in EdGB and the ingredients of Eq. (1) are now

$$
\tilde{\alpha}=-\frac{\zeta}{6}, \quad \tilde{a}=2, \quad \tilde{\gamma}=-\zeta, \quad \tilde{c}=2,
$$

where $\zeta$ is the dimensionless coupling parameter of EdGB gravity [3]. The corrections to the potential that scale as $\dot{\phi}^{2}$ are not needed because they lead to higher PN order modifications to the ppE burst model.

(v) The EdGB modifications to the orbital energy, the orbital period, and the pericenter velocity for eccentric binaries is then

$$
\begin{aligned}
\frac{\delta E_{\mathrm{EDGB}}}{E_{\mathrm{N}}} & =\frac{\zeta}{6}\left(\frac{1-e}{1+e}\right)\left(\frac{M}{r_{p}}\right)^{2}, \\
\frac{\delta T_{\mathrm{orb}}^{\mathrm{EDGB}}}{T_{\mathrm{orb}}^{\mathrm{N}}} & =-\frac{\zeta}{4}\left(\frac{1-e}{1+e}\right)\left(1+2 \sqrt{1-e^{2}}\right)\left(\frac{M}{r_{p}}\right)^{2}, \\
\frac{\delta v_{p}^{\mathrm{EDGB}}}{v_{p}^{\mathrm{N}}} & =-\frac{\zeta}{12}\left[\frac{3+e^{2}}{(1+e)^{2}}\right]\left(\frac{M}{r_{p}}\right)^{2} .
\end{aligned}
$$

These expressions correct Eq. (50) and agrees with [3] in the circular limit.

(vi) The mapping between ppE parameters and the EdGB model is now

$$
\alpha_{\mathrm{ppE}}=-\frac{\zeta}{8} \delta e+\mathcal{O}\left(\delta e^{2}\right), \quad \bar{a}_{\mathrm{ppE}}=2, \quad \beta_{\mathrm{ppE}}=-\frac{\zeta}{12}+\mathcal{O}(\delta e), \quad \bar{b}_{\mathrm{ppE}}=2
$$

where $\delta e=1-e \ll 1$. This corrects Eqs. (55) and (56). 
(vii) The time-frequency mapping of the bursts in EdGB gravity is now

$$
\begin{aligned}
t_{i}^{\mathrm{EDGB}} & =t_{i-1}+\Delta t_{(i, i-1)}^{\mathrm{GR}}\left[1-\frac{\zeta}{8} \delta e_{i}\left(\frac{M}{r_{p, i}}\right)^{2}\right], \quad f_{i}^{\mathrm{EDGB}}=f_{i}^{\mathrm{GR}}\left[1-\frac{\zeta}{12}\left(\frac{M}{r_{p, i-1}}\right)^{2}\right], \\
\delta t_{i}^{\mathrm{EDGB}} & =\delta t_{i}^{\mathrm{GR}}\left[1+\frac{\zeta}{12}\left(\frac{M}{r_{p, i-1}}\right)^{2}\right], \quad \delta f_{i}^{\mathrm{EDGB}}=\delta f_{i}^{\mathrm{GR}}\left[1-\frac{\zeta}{12}\left(\frac{M}{r_{p, i-1}}\right)^{2}\right] .
\end{aligned}
$$

This corrects Eqs. (57)-(60). These modifications to the EDGB burst model do not alter the projected constraint that can be placed on $\xi$, given by Eq. (93).

[1] N. Yunes and X. Siemens, Living Rev. Relativ. 16, 9 (2013).

[2] P. Peters, Phys. Rev. 136, B1224 (1964).

[3] N. Yunes and L. C. Stein, Phys. Rev. D 83, 104002 (2011). 